\title{
Design of optical components for terahertz/sub-terahertz imaging systems
}

\author{
A.V. Shevchik-Shekera, S.E. Dukhnin \\ V. Lashkaryov Institute of Semiconductor Physics, NAS of Ukraine, \\ 41, prospect Nauky, 03028 Kyiv, Ukraine; e-mail: annashsh82@gmail.com
}

\begin{abstract}
The possibility of using the lenses with an aspherical surface for terahertz/ sub-terahertz (THz/sub-THz) imaging systems has been considered. Diffraction-limited optical system of four identical plano-convex aspheric lenses has been obtained. Tests of the lenses have been performed on the specially made stand. These calculated and experimental data have similar values, which confirms the use of such lenses in the $\mathrm{THz} /$ sub-THz imaging systems.
\end{abstract}

Keywords: aspherical lens, THz/sub-THz imaging systems.

Manuscript received 06.04.15; revised version received 16.07.15; accepted for publication 03.09.15; published online 30.09.15.

\section{Introduction}

$\mathrm{THz} / \mathrm{sub}-\mathrm{THz}$ waves within the frequency range of 0.1 to $10 \mathrm{THz}$ are called T-rays. They occupy a large part of the electromagnetic spectrum between the infrared and microwave bands. In comparison with visible or infrared waves, $\mathrm{THz} / \mathrm{sub}-\mathrm{THz}$ radiation can penetrate into organic materials such as skin, as well as into plastics, textile, or paper. Because of low photon energy, it does not cause any damage related with ionizing radiation. $\mathrm{THz} / \mathrm{sub}-\mathrm{THz}$ waves do not penetrate into metals. These properties can be used to control material quality. It is also of great current interest for such applications as safety control, packaging inspection, semiconductor characterization, chemical composition analysis, and biomedical investigations, with great promise for spectroscopy, defense imaging, and security applications $[1,2]$.

$\mathrm{THz} / \mathrm{sub}-\mathrm{THz}$ optical components include refractive dielectric devices and metallic mirrors. Metallic mirrors are known to be achromatic and to show a very high efficiency, as actual metals in the $\mathrm{THz}$ range behave almost as ideal conductors [3]. Nevertheless, reflecting and focusing the mirrors are very sensitive to any misalignment and they do not work properly off the axis. Thus, the systems based on focusing mirrors show high geometrical aberrations, larger than the systems based on refractive lenses. Furthermore, the mirrors with large numerical aperture are heavy and have significant dimensions. On the other hand, the dielectric lenses are easy and cheaper when manufacturing. They are made of common organic plastics such as high density polyethylene (HDPE) or polytetrafluoroethylene (PTFE). They are close to achromatic ones, because the refractive index $n$ of these materials is almost constant over the whole THz/subTHz range.

\section{Designing THz/sub-THz lenses}

This paper presents the developed and manufactured aspherical lenses for $\mathrm{THz} / \mathrm{sub}-\mathrm{THz}$ imaging. These lenses have a unique profile that eliminates the 
monochromatic aberrations (e.g., spherical aberrations) and leads to an improvement in overall image quality. These lenses are small, lighter, better than similar lenses that operate only as spherical components. They are increasingly used to replace several assemblies with spherical elements, which leads to weight loss and to a more compact design. The calculated scheme for optical imaging in the THz/sub-THz range is shown in Fig. 1.

An optical system includes a $\mathrm{THz} / \mathrm{sub}-\mathrm{THz}$ source for emitting radiation. The first pair of lenses focuses light on the object, the second one - on the THz/sub$\mathrm{THz}$ detector.

The designed optical system with four identical plano-convex aspherical lenses (the lens surface is hyperboloid) is diffraction-limited. For all the calculated lenses, the focus is $70 \mathrm{~mm}$ and diameter equals $60 \mathrm{~mm}$. The focal length of lens is determined using the paraxial part of the beam. The Airy disk radius is equal to:

$r=1.22 \cdot \lambda \cdot \frac{f}{D}=1.22 \cdot 2 \mathrm{~mm} \cdot \frac{70 \mathrm{~mm}}{60 \mathrm{~mm}}=2.84 \mathrm{~mm}$,

where $f$ is the focal length, $D$ is the diameter, $\lambda$ wavelength.

The point spread function (PSF) was calculated using the optical program ZEMAX (Fig. 2). PSF expresses the normalized intensity distribution in the image plane.

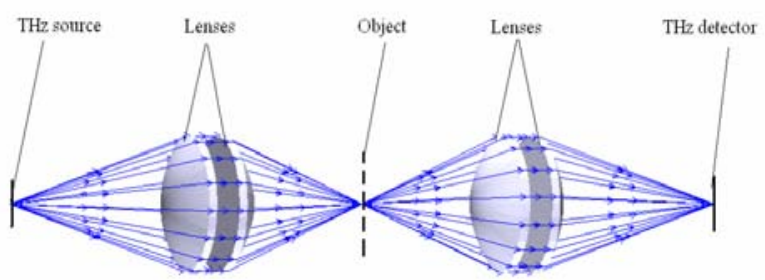

Fig. 1. The optical scheme for imaging in the THz/sub-THz range.

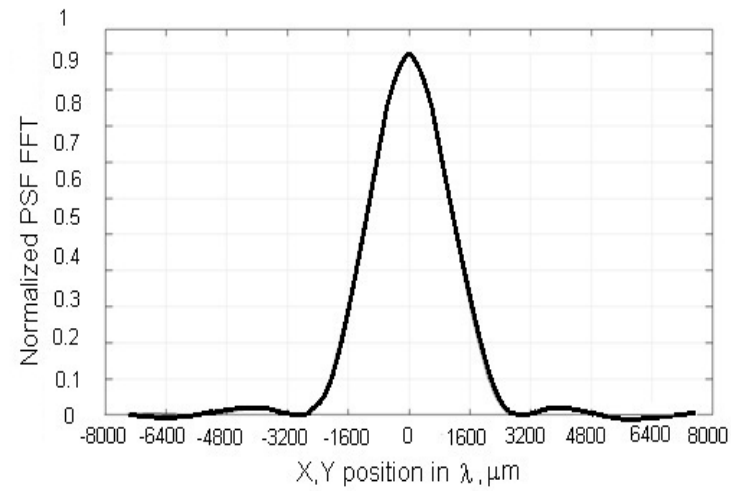

Fig. 2. PSF calculated using fast Fourier transform (FFT).

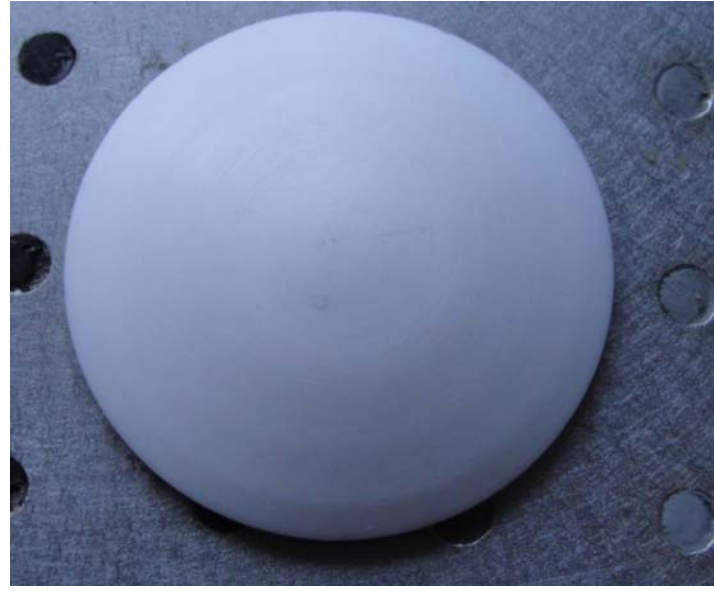

Fig. 3. The aspherical lens.

\section{Results and discussions}

Plastics PTFE with ultra-high molecular weight and refractive index of 1.43 within a wide wavelength region was selected as lens material. The lenses were milled on a computer-controlled lathe, resulting in a surface roughness is less than $30 \mu \mathrm{m}(\sim \lambda / 10)$. One of the manufactured lenses is shown in Fig. 3.

The aspherical lenses were tested on the designed stand (Fig. 4). Radiation from the THz/sub-THz source passes through the modulator with the operating frequency $140 \mathrm{GHz}$, focuses on the MCT hot electron bolometer, is amplified and recorded.

Spreading spot diagram is created by passing a sub$\mathrm{THz}$ beam through the aspheric lenses (Figs. 5 and 6).

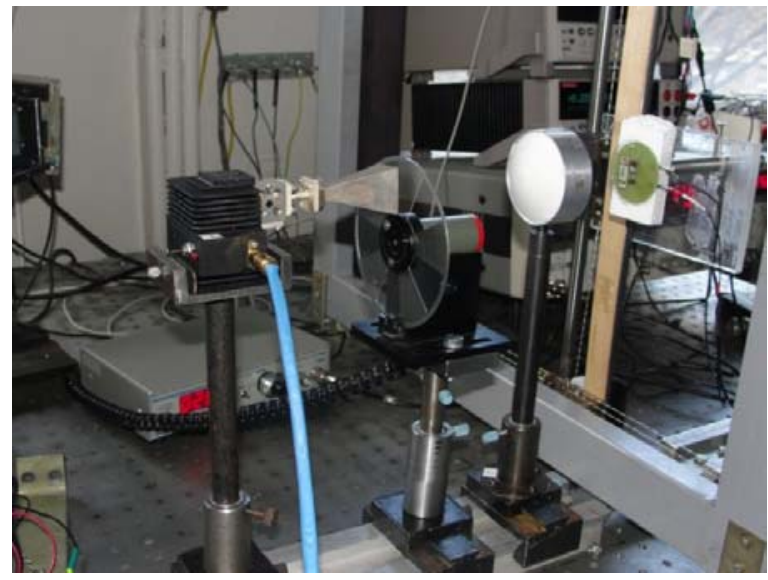

Fig. 4. Photo of the stand for testing the aspherical lenses. 


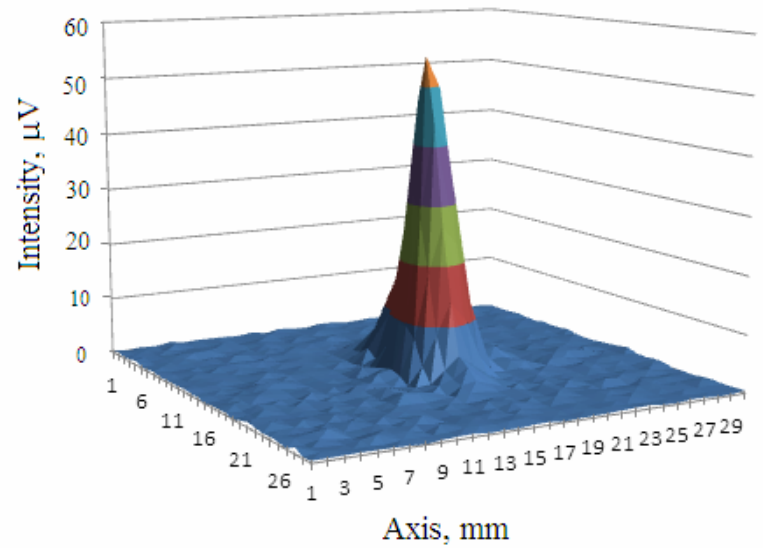

Fig. 5. 3-D lens spreading spot diagram.

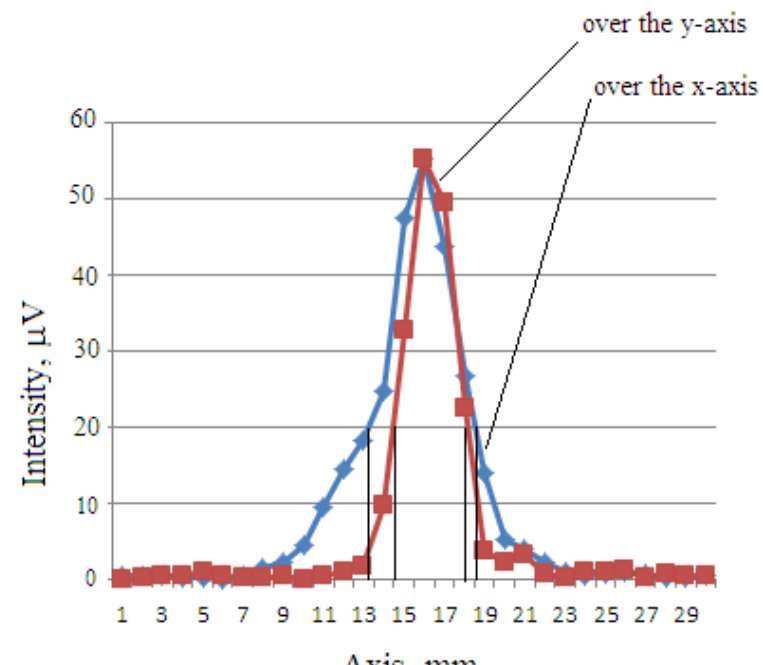

Axis, $\mathrm{mm}$

Fig. 6. Spreading spot diagram along the $x$ and $y$ axes.

A lens spreading spot diagram is the best total estimation for a quick check of image quality. It allows to quickly determine aberrations such as astigmatism, coma, spherical aberration, chromatic one and others. The central circle is a diffraction limit (Airy disk), which allows one to build the perfect objective with accurate focusing. The calculated and experimental data have similar values $\left(D_{\text {Airy-calc }}=5.6 \mathrm{~mm}, D_{\text {Airy-meas }} \approx 8 \mathrm{~mm}\right)$, respectively, this type of lenses can be used for $\mathrm{THz} / \mathrm{sub}-$ $\mathrm{THz}$ imaging systems.

\section{Conclusion}

Aspherical lenses were calculated and manufactured for $\mathrm{THz} / \mathrm{sub}-\mathrm{THz}$ imaging systems. These lenses have unique profile that eliminates monochromatic aberrations. The diffraction-limited system with four identical plano-convex aspherical lenses was obtained. All these lenses were tested on the manufactured stand. The obtained calculated and experimental data have similar values, which allows one to use these objectives in $\mathrm{THz} /$ sub-THz imaging systems.

\section{Acknowledgments}

This work is based in part on the studies with the support of NATO SfP 984,544.

\section{References}

1. F. Sizov, THz radiation sensors // Opto-Electron. Rev. 18, p. 10-36 (2010).

2. K. Kawase, Y. Ogawa, Y. Watanabe, and H. Inoue, Non-destructive terahertz imaging of illicit drugs using spectral fingerprints // Opt. Express. 11, p. $2549-2554$ (2003).

3. M. Naftaly and R. Dudley, Terahertz reflectivities of metal-coated mirrors // Appl. Opt. 50(19), p. 3201-3204 (2011). 